\begin{tabular}{|c|l|}
\hline Title & Hidden aggression in termite workers: plastic defensive behaviour dependent upon social context \\
\hline Author(s) & Ishikawa, Yuki; Miura, Toru \\
\hline Citation & $\begin{array}{l}\text { A nimal Behaviour, 83(3), 737-745 } \\
\text { https://doi.org/10.1016/.anbehav.2011.12.022 }\end{array}$ \\
\hline Issue Date & 2012-03 \\
\hline Doc URL & http://hdl.handle.net/2115/48599 \\
\hline Type & article(author version) \\
\hline File Information & AB83_3_737-745.pdf \\
\hline
\end{tabular}

Instructions for use 


\section{Hidden Aggression in Termite Workers: Plastic Defensive Behaviour Dependent upon Social Context}

Yuki Ishikawa, ${ }^{a, b}$ and Toru Miura ${ }^{a *}$

a Graduate School of Environmental Science, Hokkaido University, N10W5, Kitaku, Sapporo, 060-0810, Japan

${ }^{b}$ Present address: Graduate School of Life Sciences, Tohoku University, 2-1-1, Katahira, Aobaku, Sendai, 980-8577, Japan

* Corresponding author: miu@ees.hokudai.ac.jp, +81-01-706-4524, N10W5, Kita-ku, Sapporo, 060-0810, Japan

Word count:

Running head: Hidden aggression in termite workers 


\section{Summary}

Social insect colonies are self-organized systems that respond to changes in environmental conditions by altering the relative proportions of certain castes or individuals engaged in specific tasks. While termites are known to regulate the ratio of morphologically specialized soldiers in response to the proportion of nestmate castes, soldier-differentiation process requires a relatively long time to be accomplished. Consequently, other plastic and flexible mechanisms are considered to be employed as one of the various defensive strategies in termites. In this study, the defensive behaviours of soldiers, pseudergates (workers) and reproductives of the damp-wood termite Hodotermopsis sjostedti were quantified. When individuals of the caste were exposed to an intruder, soldiers exhibited the most vigorous defensive behaviour, followed by pseudergates and then reproductives. While the aggression levels of soldiers and reproductives were independent of the accompanying castes, pseudergate aggression was more plastic and depended on the other castes present. When paired with reproductives, pseudergates exhibited high levels of aggression toward enemies. However, pseudergate aggression levels remained low when they were paired with soldiers, suggesting that pseudergates

moderated their defensive behaviour depending on social context. Plasticity with respect to social behaviours may facilitate rapid and 
flexible responses required for colony defence.

Keywords: aggression, behavioural plasticity, soldier, colony defence, social interactions, termite 


\section{INTRODUCTION}

A variety of animal taxa modulate their behaviour and/or morphology in response to rapid changes in their environment. By changing the ratios of castes or individuals that perform specific functions within the colony, social insects exhibit plastic responses at the colony level (Wilson 1971). The regulatory mechanisms employed by a colony to respond to environmental changes without any centralized method of control are one of the major themes in sociobiology (Gordon 1996, Beshers and Fewell 2001).

The importance of colony defence in social insects is clearly apparent from the numerous defensive mechanisms that have evolved in eusocial taxa (Wilson 1975). These defence mechanisms include the use of cryptic habitats, the construction of strong and robust nests, nestmate aggregation, and the production of morphologically and/or behaviourally specialized castes. The roles of the defensive castes, such as soldiers or guards, are well defined and have been acquired independently in numerous eusocial taxa (Hermann 1984). Despite their potential contribution to colony survival, the relatively high costs associated with soldier production may result in the production of fewer workers, which take care of the soldiers in addition to their other functions like foraging, colony maintenance etc. (Rivera-Marchand et al. 2008). Thus, the proportion of soldiers or guards must be optimized 
in response to the defensive demands of the surrounding environment.

Termites are highly vulnerable to attacks by a wide variety of predators (Deligne et al. 1981). The soldier caste of termites contributes directly to colony defence by aggressively attacking intruders using specialised morphological adaptations, such as mandibles or defensive glands (Stuart 1967; Howse 1970; Deligne et al. 1981; Prestwich 1984). Optimization of soldier ratios is also expected in termites because, while they are effective for colony defence, having too many soldiers places a burden on the colony as mentioned above. The mechanisms responsible for controlling the proportion of soldiers within a colony have been investigated previously in classical studies, which suggested that the presence of soldiers inhibits the differentiation of new soldiers from workers (or pseudergates), while the presence of reproductive castes or nymphs accelerates it (Castle 1934; Miller 1942; Springhetti 1970; Nagin 1972; Lentz 1976; Haverty \& Howerd 1981; Lefeuve \& Bordereau 1984). Although encounters with enemies can be sudden and unpredictable, the response of the colony with respect to defensive task allocation through controlling caste ratios is limited by the fact that soldier differentiation requires 15 to 30 days to complete (Ogino et al. 1993, Cornette et al. 2008). Consequently, the development of defence systems that are more flexible and rapid would be expected. 
Workers of social insects are the primary workforce in a colony, performing tasks such as foraging, brood care, nest maintenance, and sometimes colony defence. These workers can respond to changes in the surrounding environment independently of their age or morphology (Gordon 1996). Indeed, in some ant species, workers modify their aggression without undergoing any morphological modifications depending on the presence of other nestmates (Tanner 2006; Tanner 2008; Tanner and Alder 2009). Therefore, we particularly focused on termite workers as the potential defensive force within a colony when soldiers were absent. Although worker termites are known to defend their colonies, primarily through actions involving the construction and repair of nest structures, direct defensive behaviours (e.g. biting) have only been reported in soldier-less species or in species with soldiers that have well-developed frontal glands and reduced mandibles (Stuart 1967; Eisner et al 1976; Deligne et al. 1981; Traniello \& Beshers 1985).

In this study, we investigated the context-dependent plasticity of defensive behaviours in soldiers, pseudergates and reproductives of the damp-wood termite Hodotermopsis sjostedti. The focal termite species is considered to retain several primitive eusocial characteristics (Thorne and Traniello 2003), such as biting-type soldiers and a linear caste-differentiation pathway (Fig. 1a) (Miura et 
al. 2000, Miura et al. 2004). In this species, true workers are absent, and elder juveniles, which are referred to as pseudergates (etymologically "false workers"), perform worker tasks. Pseudergates are socially active and potentially capable of developing into imagos (Grassé \& Noirot 1947; Noirot 1985; Thorne 1996; Parmentier \& Roisin 2003). In H. sjostedti, after hatching from eggs, larvae undergo six moults before developing into pseudergates (Miura et al. 2000, Miura et al. 2004). While pseudergates are totipotent and capable of differentiating into reproductives or sterile soldiers, most remain pseudergates through stationary moults (Figure 1a, Miura et al. 2000, Miura et al. 2004).

We initially examined differences in defensive responses among individual soldiers, pseudergates and reproductives when exposed to the ant Formica japonica. We then examined whether the caste-specific tendencies of the defensive response were altered by the presence of nestmates. Since defensive tasks (e.g. encountering enemies) are usually restricted to the peripheral regions of a nest, caste localization within the nest is also important for defensive task allocation. Therefore, this study also examined the positioning and behavioural responses of the castes at the time of nest opening in the presence of other nestmates. 


\section{MATERIALS AND METHODS}

\section{Insects}

Colonies of the damp-wood termite Hodotermopsis sjostedti were sampled from decaying wood in evergreen forests on Yakushima Island in Kagoshima Prefecture, Japan. Colonies were maintained in the laboratory as stock at approximately $25^{\circ} \mathrm{C}$ under constant darkness. Soldiers, neotenics and pseudergates were used for the behavioural experiments. As in previous studies (Miura et al 2000, Miura et al. 2004), seventh-instar larvae were regarded as pseudergates in this study. This species is considered to have two reproductive castes, primary reproductives and neotenics (supplementary or secondary reproductives) (Miura et al. 2000). As it is common in lower termites, most mature $H$. sjostedti colonies in nature do not contain a pair of primary reproductives, but have dozens of neotenics instead (Matsumoto \& Hirono 1985). In this study, therefore, neotenics were regarded as being representatives of the reproductive caste.

\section{Experimental set up}

To compare defensive behaviours among castes, individual termites were introduced into a plastic Petri dish (diameter: $60 \mathrm{~mm}$ ) which was placed in an arena $(14 \mathrm{~cm} \times 10 \mathrm{~cm})$ in a plastic enclosure. The dish, 
which was considered to represent an artificial nest, was lined with moist filter paper and had an opening representing the main entrance (hereafter referred to as the gate), which could easily be opened or closed by rotating the lid of the dish. Once inside the artificial nest, the termite was exposed to an ant, which was placed on the end of a toothpick and positioned inside the gate (figure 1b). The tip of toothpick was attached to the dorsal thorax of the ant with adhesive, so that it could move its legs freely.

Comparisons of aggressive responses among castes in response to enemy invasion

We compared aggressive responses among castes in response to enemy invasion under isolated conditions in which individual termites were introduced into a nest (Fig. 1b, top). After being allowed to acclimatise in the nest for $15 \mathrm{~min}$, the gate was opened and a living ant (Formica japonica), placed on the tip of a wooden toothpick, was positioned inside the open gate. The behaviour of the individual termite was then recorded using a digital video camera recorder (Handycam DCR-PC5, Sony Corporation, Japan) for 3 min after encountering the ant. Defensive behaviours were defined as (i) biting, if the termite bit the ant, or (ii) assuming an attack posture, if the termite directed its head toward the ant in the area delimited by the dotted line in Fig. $1 \mathrm{~b}$. 
The number of times each termite opened and closed its mandibles, and the amount of time the termite assumed an attack posture were measured and used as indexes of biting and attack posture, respectively. Both sexes of each caste were used in the behavioural assays. However, since the sex of the termite did not affect the extent of the behavioural response (Biting: $U=288, N_{\text {male }}=35, N_{\text {female }}=11$, $P>0.05$, Attack posture: $U=218.5, \quad N_{\text {male }}=35, \quad N_{\text {female }}=11, P>0.05$, Mann-Whitney $U$ test), we did not discriminate between sexes when analyzing the data. The number of experiments and colonies analysed are shown in Table1.

Next, to clarify whether any caste-specific tendencies of the defensive response existed with respect to the presence of nestmates, behavioural observations were conducted under paired conditions (Fig. 1b, middle). Under paired conditions, the behavioural responses of two termite individuals that had been placed inside the nest for 15 min and exposed to an ant at the gate were recorded for 3 min after one of the termites had encountered the ant intruder. The paired individuals were selected from the same colony. Numbers of trials and colonies are described in Table1.

Differences in the biting frequency or the duration of an attack posture were assessed statistically using the Kruskal-Wallis test. When the overall Krukal-Wallis tests were significant, the Steel-Dwass tests 
were employed to evaluate the significant differences between each individual category. Because the analyses of the defensive behaviours included multiple comparisons, the cut-off point for rejection of the null hypothesis in each test was determined by the procedure of Guo and Rao (2008) to control the false discovery rate at lower than $5 \%$. The approach is a modified "step-down" procedure of Benjamini \& Hochberg (1995), and can be applied irrespective of the strength of correlation among the tests. The control of $P$ values by the original method of Benjamini \& Hochberg (1995), which can be applied under the assumption of independence or positive correlation, gave same results. Alpha levels were calculated for each statistical test of each experiment, and were stated in Table2.

\section{Comparison of aggressive behaviour in response to opening the} nest

Under both natural and laboratory conditions, when termite nests are exposed to open air, all colony members respond to the disturbance (some escape to the centre of the nest while others exhibit an aggressive response) (Eisner et al. 1976). We therefore examined the responses of the termite castes to nest opening in terms of the positions they assume and their behaviours.

Five pseudergates were introduced into a nest together with 
either five nestmate soldiers or five nestmate neotenics ( $n=11$ groups, Fig. 1b, bottom). Five colonies were used for groups of pseudergates and soldiers, and two colonies were used for groups of pseudergates and neotenics. In preliminary studies, 15 min of acclimatization was not sufficient to keep the termites inside the opened nest. Consequently, we left the ten individuals to be tested in the nest overnight before initiating the experiments. All of the termites were marked with oil paint to facilitate individual identification. After placing the nest in the arena for $15 \mathrm{~min}$, the gate was opened and the behaviours of all of the termites in the dish were recorded with the digital video camera for 10 min. In this experiment, the observed defensive behaviours were defined as (i) head banging, considered as an alarm signal (Howse 1964), if the termite repeatedly beat its head on the ground, (ii) guarding, if the termite directed its head toward the gate in the region delineated by the dotted line in Fig. 1b (bottom), and (iii) patrolling, if the termite walked around the outside the Petri dish in the arena. In addition, examples of non-defensive behaviours were also defined, including (iv) walking, if termites walked around in the dish, (v) gnawing, if termites gnawed the filter paper or dish wall, and (vi) grooming, if the termites groomed other individuals.

Differences in each defensive behaviours and non-defensive behaviours among all individual categories (neotenics with 
pseudergates, pseudergates with neotenics, pseudergates with soldiers, and soldiers with pseudergates) were assessed statistically using the Kruskal-Wallis test. When the Krukal-Wallis test for the behaviour was significant, the Steel-Dwass tests were employed to evaluate the significant differences between the individual categories. Because the analyses included multiple comparisons, the cut-off point for rejection of the null hypothesis in each test was determined by the procedure of Guo \& Rao (2008) to control the false discovery rate at lower than 5\%. Alpha levels were calculated independently for each statistical test of each experiment, and were stated in Table2. 


\section{RESULTS}

Comparison of aggressive responses toward intruders under isolated conditions

We first compared the defensive behaviours (biting and assuming an attack posture) of castes in response to enemy invasion under isolated conditions (Fig. 1b, top). As in nature, soldiers exhibited defensive behaviours more frequently than the other castes (Fig. 2, Table2). After encountering an ant, the soldiers bit it repeatedly until it was dead; the frequency of this defensive behaviour then decreased. Soldiers rarely exhibited an escape response after encountering an ant. Pseudergates also exhibited several defensive behaviours under isolated conditions (Fig. 2). Upon encountering an ant, pseudergates often bit the ant several times before escaping to give an attack posture that was shorter in duration than that observed in soldiers (Fig. 2b); although less effective than soldiers, most of the pseudergates that attacked the ants either killed or injured the intruder (Table 1). Neotenics exhibited the weakest defensive responses of all of the castes tested, both in terms of biting and the assumption of an attack posture (Fig. 2, Table2).

Comparison of aggressive responses toward intruders under paired conditions

We then clarified whether the caste-specific tendencies of defensive 
responses were affected by the presence of nestmates under paired conditions (Fig. 1b, middle). The results showed that soldiers were consistently aggressive while neotenics were seldom aggressive, and that these levels of aggression were generally maintained regardless of which castes are placed together (Fig.3). Duration of attack postures of neotenics paired with soldiers tend to be higher than when neotenics were paired with neotenics (Fig. 3, Table2), this is because neotenics spent more time with the ant killed by soldiers, assuming an attack posture.

However, pseudergates dramatically altered their defensive behaviour depending on which caste they were paired with (Fig. 3, Table2). When paired with soldiers, the frequency of defensive behaviours exhibited by pseudergates was lower than when paired with pseudergates or neotenics, and was similar in intensity to that exhibited by neotenics (Fig. 3, Table2). On the other hand, the frequency of defensive responses in pseudergates paired with a neotenics was higher than when pseudergates were paired with soldiers or pseudergates (Fig. 3, Table2). Indeed, when paired with neotenics, the levels of aggression among pseudergates did not differ significantly from that exhibited by soldiers (Fig. 3, Table2). The escape behaviour of pseudergates decreased when paired with a neotenics (data not shown). 


\section{Comparisons of aggression in response to nest opening}

We then compared the caste localization within the nest and defensive behaviour of castes under conditions of nest disturbance (Fig. $1 b$, bottom). In an experimental group consisting of five neotenics and five pseudergates, the pseudergates positioned themselves near the gate and exhibited more aggression than neotenics (Fig. 4, A1, Table2). In an experimental group consisting of five pseudergates and five soldiers, soldiers positioned themselves near the gate and exhibited higher frequencies of defensive behaviour than the pseudergates (Fig. 4, A1, Table2). Similar to the direct behavioural responses against an intruder (Fig. 3), the positioning and defensive response of pseudergates to nest disturbance seemed to be affected by the coexisting castes. The frequency of the head-banging (alarm) behaviour and the localization (guarding) in pseudergates grouped with neotenics tended to be higher than when they were grouped with soldiers (Fig. 4, A1, Table2). 


\section{DISCUSSION}

Our results showed that pseudergates possess the potential to attack enemies by altering their behaviour in a context-dependent manner. When exposed to an intruding ant, pseudergates exhibited relatively low levels of aggression in the presence of soldiers, but very high levels of aggression in the presence of neotenics (Fig. 3, 5). A similar result was observed in the manner in which pseudergates locate themselves within the nest in response to a nest disturbance (Fig. 4, 5). Based on these findings, pseudergates under natural conditions are thought to retreat deep inside the nest if there are soldiers and no reproductives around them, or to remain on-site and attack intruders if there are reproductives and no soldiers. A similar finding was suggested by Roision et al. 1990, who found that defensive responses in workers of Nasutitermes princeps also decreased in the presence of soldiers. Since these behavioural changes can occur within minutes after the defence demands of a colony have changed, a flexible and rapid defensive strategy can be achieved through the behavioural plasticity of workers (or pseudergates). Context-dependence was also observed in the alarm response, i.e. more frequent head banging of pseudergates in the absence of soldiers. Although a behavioural response to such an alarm signal has not yet clearly been demonstrated to date, such signals are considered to act as a trigger for the recruitment of 
additional soldiers (and/or workers and pseudergates) to the sites of reproductives. Thus, the plastic defensive response of workers or pseudergates may play essential roles in the optimization of colony defence by compensating for the relatively slow responsiveness in caste differentiation (Fig. 5).

The findings of this study also suggest that pseudergates possess the ability to recognize the castes in their vicinity. Since the compound eyes and optic lobes of workers and pseudergates are markedly reduced, it is unlikely that they discriminate castes by visual cues. Recent studies on caste-specific chemicals secreted from exocrine glands or epithelia (Miura et al. 1999; Hanus et al. 2009; Liebig et al. 2009; Weil et al. 2009; Korb et al. 2009) suggest that either odour or contact signals act as caste-recognition cues. Sound is another potential candidate signals for distinguishing between castes, as soldiers exhibit higher head banging frequencies than pseudergates and neotenics (Fig. 4), and the actual sounds produced by soldiers may also differ from those produced by pseudergates and neotenics (Howse 1964). To more accurately clarify these different caste-recognition cues, experiments using chemicals or audio stimuli in isolation will be required.

Since the presence of castes affects both the behaviours and development of pseudergates (Castle 1934; Miller 1942; Springhetti 
1970; Nagin 1972; Lentz 1976; Haverty \& Howerd 1981; Lefeuve \& Bordereau 1984, the present study), it is likely that some physiological links exist between changes in aggression and the regulation of soldier differentiation (Fig. 5). It is well known that juvenile hormone (JH) plays an important role in the caste determination of termites, with exposure to high $\mathrm{JH}$ levels during inter-moult periods triggering the differentiation of workers into soldiers, while exposure to low JH levels is considered to induce alate differentiation (Nijhout \& Wheeler 1982; Cornette et al. 2008). Recent studies have suggested that insect aggression is also affected by $\mathrm{JH}$; for example, high $\mathrm{JH}$ titres cause increased male aggression in Lobster cockroaches Nauphoeta cinerea (Kou et al. 2009). This suggests that in termites, the recognition of nestmates may possibly alter $\mathrm{JH}$ levels in pseudergates, resulting in both changes in aggression and caste determination (Fig. 5).

Since almost all extant termite species possess distinctive soldier castes (the loss of a soldier caste in some termites is considered to have occurred secondarily), the evolutionary process by which the morphologically and behaviourally unique soldier caste has been originated (as an evolutionary novelty) is difficult to postulate. Deligne (1981) suggested that the soldier caste was derived from workers, but the evolutionary process through which highly differentiated soldiers have originated from less aggressive and undifferentiated workers 
remains unclear. In this study, pseudergates exhibited aggression comparable to soldiers in certain situations. If the workers or pseudergates (i.e. helpers) of ancestral termite species exhibited a similar behavioural plasticity, then it can be hypothesized that the plastic nature of aggressive behaviour may have varied among genetically distinct colonies or populations. This plastic response may then gradually have become fixed as morphological specialization occurred, either subsequently or concurrently, in response to predation pressure. This would satisfy the classic idea that behavioural plasticity is the first step involved in evolutionary change, and that this change is then succeeded by morphological specialization (Baldwin 1896 \& 1902; Wcislo 1989). To verify this scenario, further studies into the genetic variation underlying plastic characters need to be conducted in the future. 


\section{REFERENCES}

Baldwin, J. M. 1896. A new factor in evolution. American Naturalist, 30, 441-451.

Baldwin, J. M. 1902. Development and Evolution. New York: Macmillan. Benjamini, Y. \& Hochberg, Y. 1995. Controlling the False Discovery Rate a Practical and Powerful Approach to Multiple Testing. Journal of the Royal Statistical Society Series B-Methodological, 57, 289-300.

Beshers, S. N. \& Fewell, J. H. 2001. Models of division of labor in social insects. Annual Review of Entomology, 46, 413-440.

Castle, G. 1934. The damp-wood termites of the western United States, genus Zootermopsis (formerly, Termopsis) In: Termites and Termite Control (Ed. by Kofoid, C.), pp. 273-310. Berkeley: University of California Press. Cornette, R., Gotoh, H., Koshikawa, S. \& Miura, T. 2008. Juvenile hormone titers and caste differentiation in the damp-wood termite Hodotermopsis sjostedti (Isoptera, Termopsidae). Journal of Insect Physiology, 54, 922-930.

Deligne, J., Quennedey, A. \& Blum, M. 1981. The enemies and defence mechanisms of termites. In: Social insects (Ed. by Hermann, H.), pp. 1-76. London: Academic Press.

Eisner, T., Kriston, I. \& Aneshansley, D. J. 1976. Defensive behavior of a Termite (Nasutitermes exitiosus). Behavioral Ecology and Sociobiology, 1, 83-125. 
Grassé, P. P. \& Noirot, C. 1947. Le polymorphisme social du termite á cou jaune (Calotermes flavicollis F.). Les faux-ouvriers ou pseudergates et les mues régressives. Les Comptes Rendus de l'Académie des Sciences de Paris, 224, 219-221

Gordon, D. M. 1996. The organization of work in social insect colonies. Nature, 380, 121-124.

Guo, W. G. \& Rao, M. B. 2008. On control of the false discovery rate under no assumption of dependency. Journal of Statistical Planning and Inference, 138, 3176-3188.

Hanus, R., Vrkoslav, V., Hrdý, I., Cvacka, J. \& Sobotnik, J. 2009. Beyond cuticular hydrocarbons: evidence of proteinaceous secretion specific to termite kings and queens. Proceedings of the Royal Society B: Biological Sciences, 277, 995-1002.

Haverty, M. \& Howard, R. 1981. Production of soldier proportions by laboratory experimental groups of Reticulitermes flavipes (Koller) and Reticulitermes virginicus (Banks) (Isoptera: Rhinotermitidae). Insectes Sociaux, 28, 32-39.

Hermann, H. e. 1984. Defensive Mechanisms In Social Insects. New York: Praeger Publishers.

Howse, P. E. 1964. The significance of the sound produced by the termite Zootermopsis angusticollis (Hagen). Animal Behaviour, 12, 284-300 
Howse, P. E. 1970. Termites: A Study In Social Behavior. London: Hutchinson University Library.

Korb, J., Weil, T., Hoffmann, K., Foster, K. R. \& Rehli, M. 2009. A gene necessary for reproductive suppression in termites. Science, 324, 758.

Kou, R., Chou, S. Y., Chen, S. C. \& Huang, Z. Y. 2009. Juvenile hormone and the ontogeny of cockroach aggression. Hormones and Behavior, 56, $332-338$.

Lefeuve, P. \& Bordereau, C. 1984 . Soldier formation regulated by a primer pheromone from the soldier frontal gland in a higher termite, Nasutitermes-Lujae. Proceedings of the National Academy of Sciences of the United States of America-Biological Sciences, 81, 7665-7668.

Lenz, M. 1976. The dependence of hormone effects in termite caste determination on external factors. In: Phase and Caste Determination in Insects (Ed. by Lüscher, M.). Oxford and New York: Pergamon Press. Liebig, J., Eliyahu, D. \& Brent, C. S. 2009. Cuticular hydrocarbon profiles indicate reproductive status in the termite Zootermopsis nevadensis. Behavioral Ecology and Sociobiology, 63, 1799-1807.

Matsumoto, T. \& Hirono, Y. 1985. On the caste composition of a primitive termite Hodotermopsis japonicus Holmgren (Isoptera, Termopsidae). Scientific Papers of the College of Arts and Sciences, The University of Tokyo, $35,211-216$. 
Miller, E. 1942. The problem of castes and caste differentiation in Prorhinotermes simplex Hagen. Bulletin of the University of Miami, 15, 1-27. Miura, T., Kamikouchi, A., Sawata, M., Takeuchi, H., Natori, S., Kubo, T. \& Matsumoto, T. 1999. Soldier caste-specific gene expression in the mandibular glands of Hodotermopsis japonica (Isoptera : Termopsidae). Proceedings of the National Academy of Sciences of the United States of America, 96, 13874-13879.

Miura, T., Hirono, Y., Machida, M., Kitade, O. \& Matsumoto, T. 2000. Caste developmental system of the Japanese damp-wood termite Hodotermopsis japonica (Isoptera : Termopsidae). Ecological Research, 15, 83-92.

Miura, T., Koshikawa, S., Machida, M. \& Matsumoto, T. 2004. Comparative studies on alate wing formation in two related species of rotten-wood termites: Hodotermopsis sjostedti and Zootermopsis nevadensis (Isoptera, Termopsidae). Insectes Sociaux, 51, 247-252.

Nagin, R. 1972. Caste determination in Neotermes jouteli (Banks). Insectes Sociaux, 19, 39-61.

Nijhout, H. F. \& Wheeler, D. E. 1982. Juvenile-Hormone and the Physiological-Basis of Insect Polymorphisms. Quarterly Review of Biology, 57, 109-133. 
Noirot, C. 1985. Pathways of Caste Development in the Lower Termites. In: Caste Differentiation in Social Insects (Ed. by Watson, J., Okot-Kotber, B. \& Noirot, C.), pp. 41-58. Oxford: Pergamon Press.

Ogino, K., Hirono, Y., Matsumoto, T. \& Ishikawa, H. 1993. Juvenile hormone analog, S-31183, causes a high-Level induction of presoldier differentiation in the Japanese damp-wood termite. Zoological Science, 10, 361-366.

Parmentier, D. \& Roisin, Y. 2003. Caste morphology and development in Termitogeton nr. planus (Insects, Isoptera, Rhinotermitidae). Journal of Morphology, 255, 69-79.

Prestwich, G. D. 1984. Defense mechanisms of termites. Annual Review of Entomology, 29, 201-232.

Rivera-Marchand, B., Giray, T. \& Guzman-Novoa, E. 2008. The cost of defense in social insects: insights from the honey bee. Entomologia Experimentalis Et Applicata, 129, 1-10.

Roisin, Y., Everaerts, C., Pasteels, J. M. \& Bonnard, O. 1990. Caste-dependent reactions to soldier defense secretion and chiral alarm/recruitment pheromone in Nasutitermes princeps. Journal of Chemical Ecology, 16, 2865-2875.

Springhetti, A. 1970. Influence of the king and the queen on the differentiation of soldiers in Kalotermes flavicollis Fabr. (Isoptera). Monitore Zoologico Italiano, 4, 99-105. 
Stuart, A. M. 1967. Alarm, defense, and construction behavior relationships in termites(Isoptera). Science, 156, 1123-1125.

Tanner, C. J. 2006. Numerical assessment affects aggression and competitive ability: a team-fighting strategy for the ant Formica xerophila. Proceedings of the Royal Society B-Biological Sciences, 273, 2737-2742. Tanner, C. J. 2008. Aggressive group behaviour in the ant Formica xerophila is coordinated by direct nestmate contact. Animal Behaviour, 76, 1335-1341. Tanner, C. J. \& Adler, F. R. 2009. To fight or not to fight: context-dependent interspecific aggression in competing ants. Animal Behaviour, 77, 297-305.

Thorne, B. L. 1996. Termite terminology. Sociobiology, 28, 253-263. Thorne, B. L. \& Traniello, J. F. A. 2003. Comparative social biology of basal taxa of ants and termites. Annual Review of Entomology, 48, 283-306.

Traniello, J. F. A. \& Beshers, S. N. 1985. Species-specific alarm recruitment responses in a neotropical termite. Naturwissenschaften, $\mathbf{7 2}$, 491-492.

Wcislo, W. T. 1989. Behavioral environments and evolutionary change. Annual Review of Ecology and Systematics, 20, 137-169.

Weil, T., Hoffmann, K., Kroiss, J., Strohm, E. \& Korb, J. 2009. Scent of a queen-cuticular hydrocarbons specific for female reproductives in lower termites. Naturwissenschaften, 96, 315-319. 
Wilson, E. O. 1971. The Insect Societies. Cambridte, Massachusetts, and London, England: The Belknap Press of Harvard University Press.

Wilson, E. O. 1975. Sociobiology: The New Synthesis. Cambridge: Harvard University Press. 


\section{Appendix}

A1
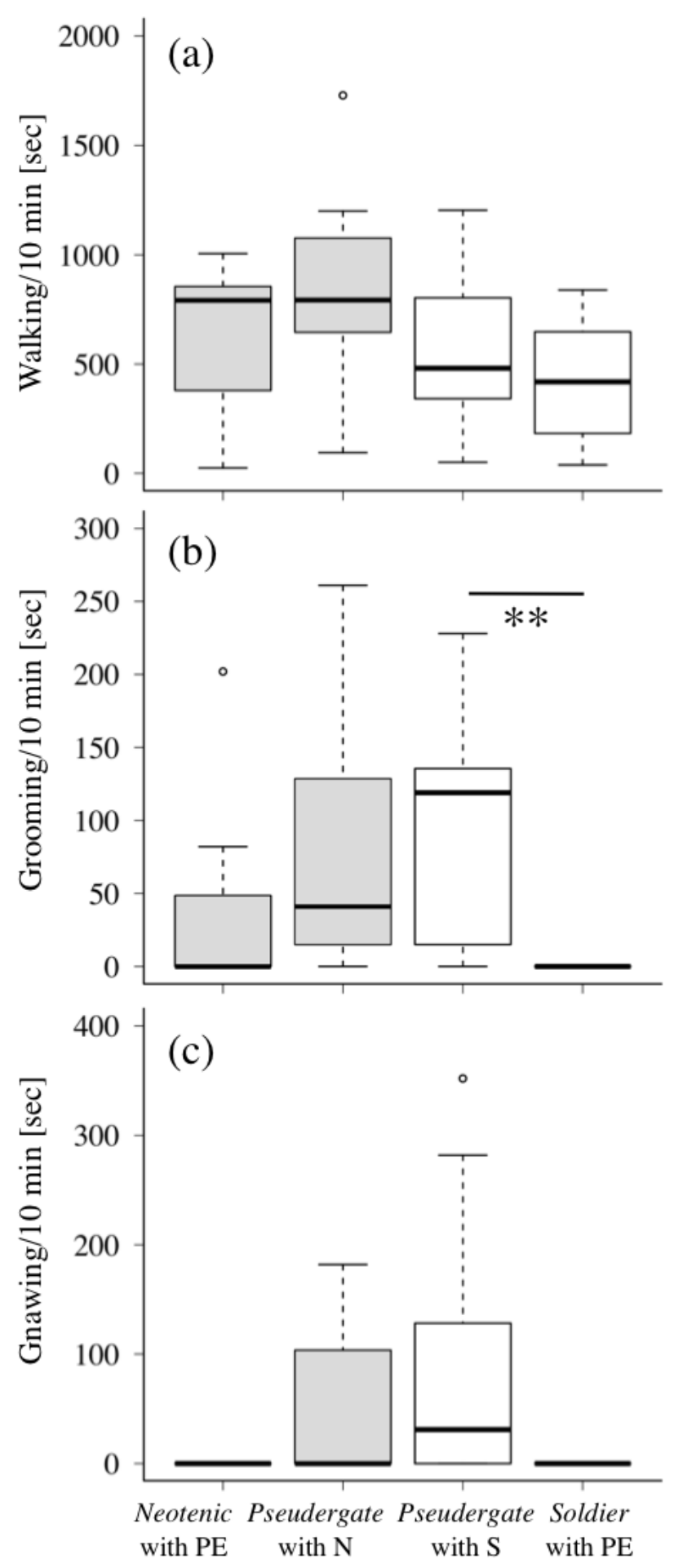


\section{Figure captions}

Figure 1. (a) Caste differentiation in Hodotermopsis sjostedti. Larvae undergo six moults to develop into pseudergates, some of which then differentiate into sterile soldiers or reproductive castes, such as alates and neotenics. (b) Experimental designs for the quantification of defensive behaviour. To quantify the behavioural response against enemy intrusion (Figs 2 and 3), biting frequency and duration of attack posture under isolated or paired conditions were recorded for $3 \mathrm{~min}$ while exposed to an ant (top, middle). For quantitatively assessing the behavioural response against nest opening (Fig. 4), head banging, guarding, patrolling, walking, gnawing, grooming were recorded for a 10 min period following opening of the gate (bottom).

Figure 2. Differences in the defensive behaviour of castes in response to enemy intrusion under isolated conditions. Soldiers exhibited the most aggressive behaviours, consisting of (a) biting and (b) assuming an attack posture. Pseudergates were the next most aggressive caste and neotenics were the least aggressive. Box plots show the median, upper and lower quartiles and range. Whiskers indicate minima and maxima, and circles indicate outliers' values, respectively. Different letters indicate statistical significance $(p<0.05$, Kruskal-Wallis test 
followed by Steel-Dwass test).

Figure 3. Differences in the defensive behaviour of castes in response to enemy intrusion under paired conditions. While soldiers and neotenics exhibited constant levels of biting (a) and attack posture (b) irrespective of the accompanying castes, pseudergates altered their aggression dramatically depending on the co-existing castes. Box plots show the median, upper and lower quartiles and range. Whiskers indicate minima and maxima, and circles indicate outliers' values, respectively. Different characters indicate statistical significance ( $p<0.05$, Kruskal-Wallis test followed by Steel-Dwass test).

Figure 4. Differences in the defensive behaviour of castes in response to nest disturbance (nest opening). Neotenics were less aggressive than pseudergates in a group consisting of five pseudergates and five neotenics. Soldiers exhibited more frequent defensive behaviours (head banging and guarding) than pseudergates in an experimental group consisting of five pseudergates and five soldiers. When comparing pseudergate behaviour between the different conditions (i.e. pseudergates with soldiers vs. pseudergates with neotenics), pseudergates grouped with neotenics tended to be more aggressive than pseudergates grouped with soldiers. Box plots show the median, 
upper and lower quartiles and range. Whiskers indicate minima and maxima, and circles indicate outliers' values, respectively. Asterisks indicate statistical significance $(* P<0.05, * * P<0.01$, Kruskal-Wallis test followed by Steel-Dwass test).

Figure 5. Regulation mechanism of colony defence in the focal termite species based on the results of this study. Pseudergates are plastic with respect to both aggression (defensive behaviour) and developmental fates, while soldiers and neotenics exhibit stable behaviour and are developmentally terminal. The presence of soldiers represses both soldier differentiation and worker aggression, while the presence of neotenics promotes soldier development.

Fig. A1 Differences in castes behaviours in response to nest opening. Non-defensive behaviours (Walking, Grooming, Gnawing) were not frequent in soldiers, while defensive behaviours (head banging, guarding and patrolling) were more frequent in soldiers than in pseudergates (Fig. 4), which were more aggressive than neotenics. When comparing pseudergate behaviours between the different conditions (i.e. pseudergates with soldiers vs. pseudergates with neotenics), pseudergates with neotenics exhibited higher levels of aggression than pseudergates with soldiers (see also Fig, 4). Box plots 
show the median, upper and lower quartiles and range. Asterisks indicate statistical significance $(* P<0.05, * * P<0.01$, Kruskal-Wallis test followed by Steel-Dwass test) 
(a)

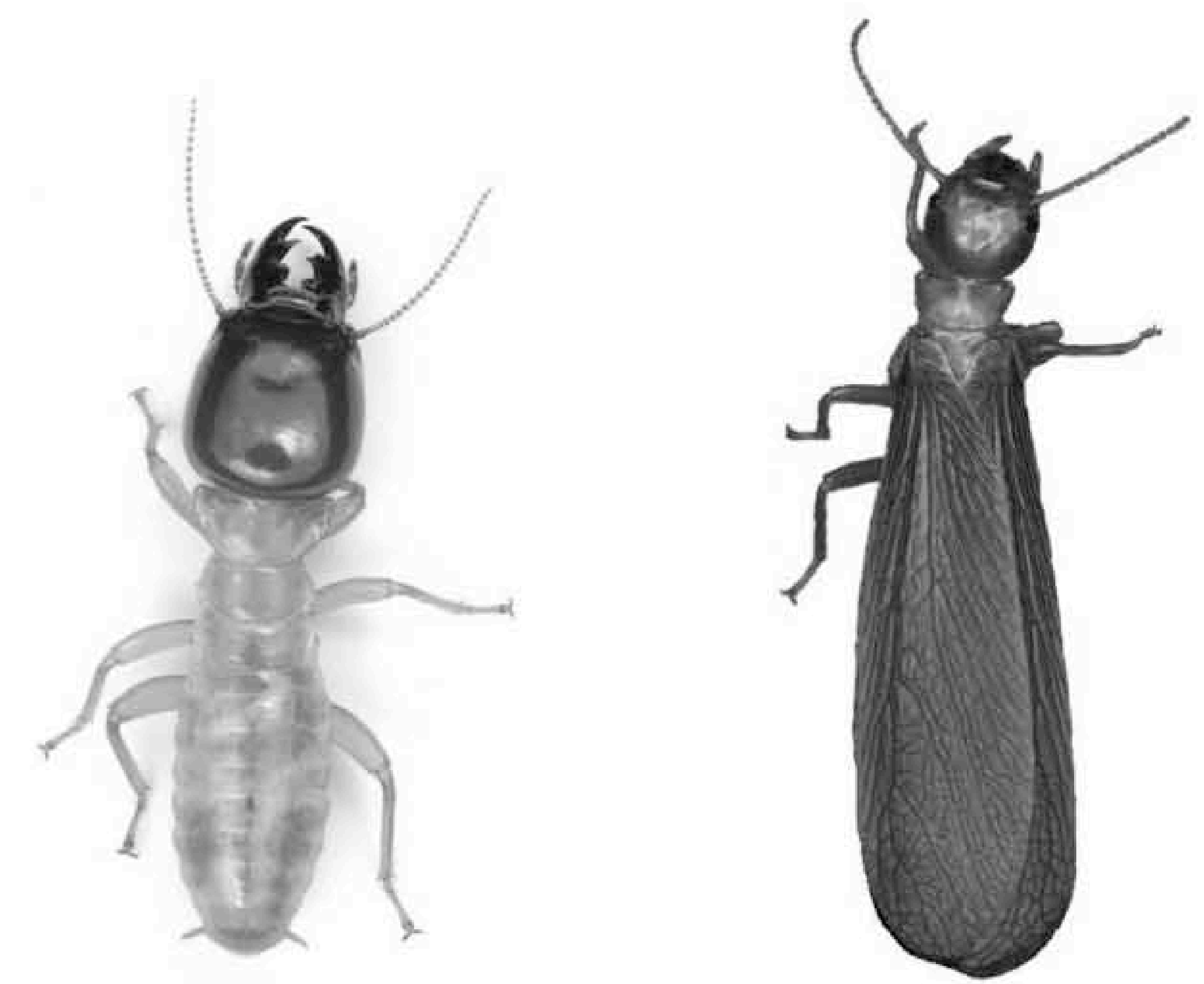

Soldier

$\mathbf{n}$

Alate
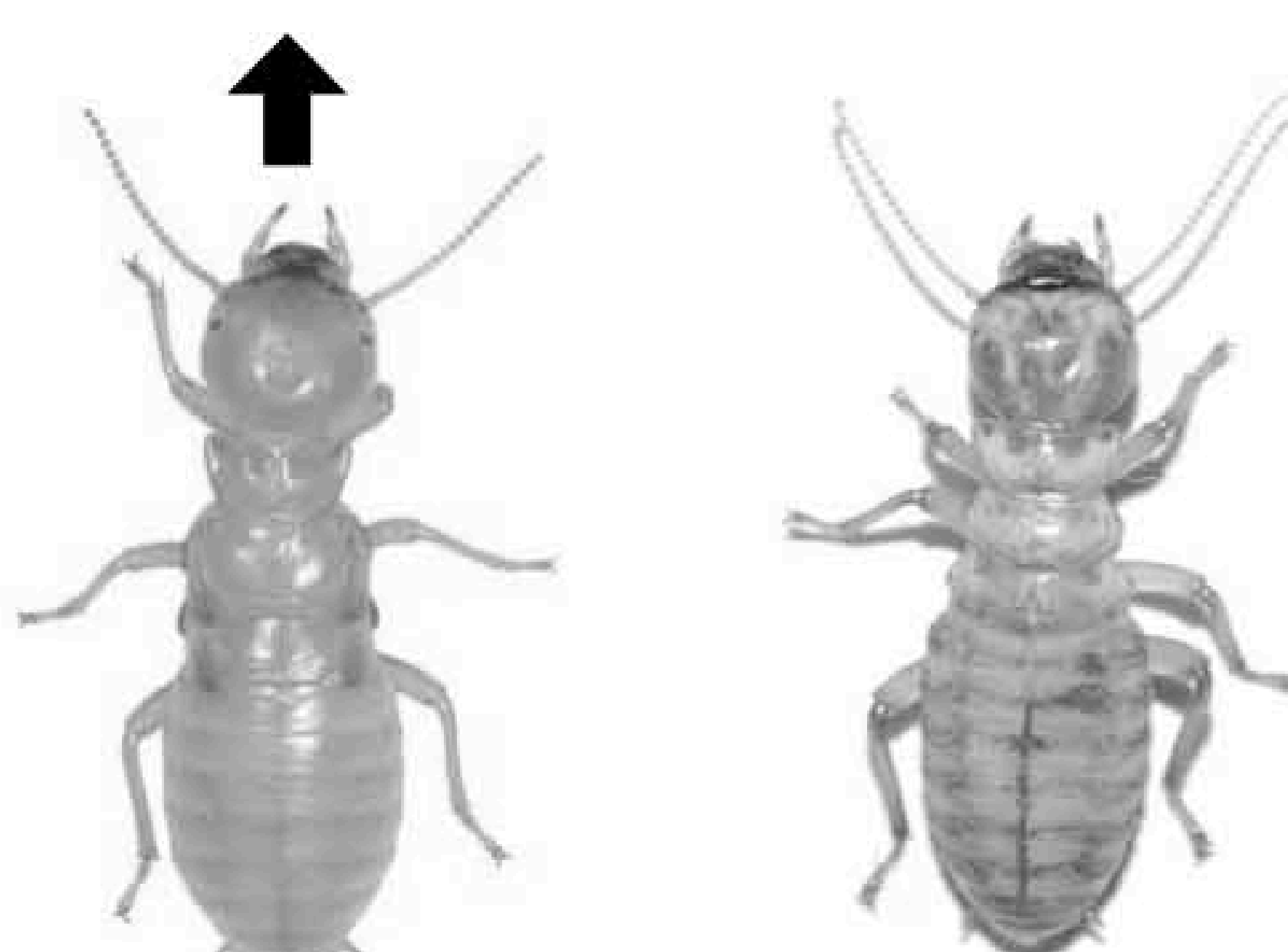

Presoldier Nymph

Neotenic

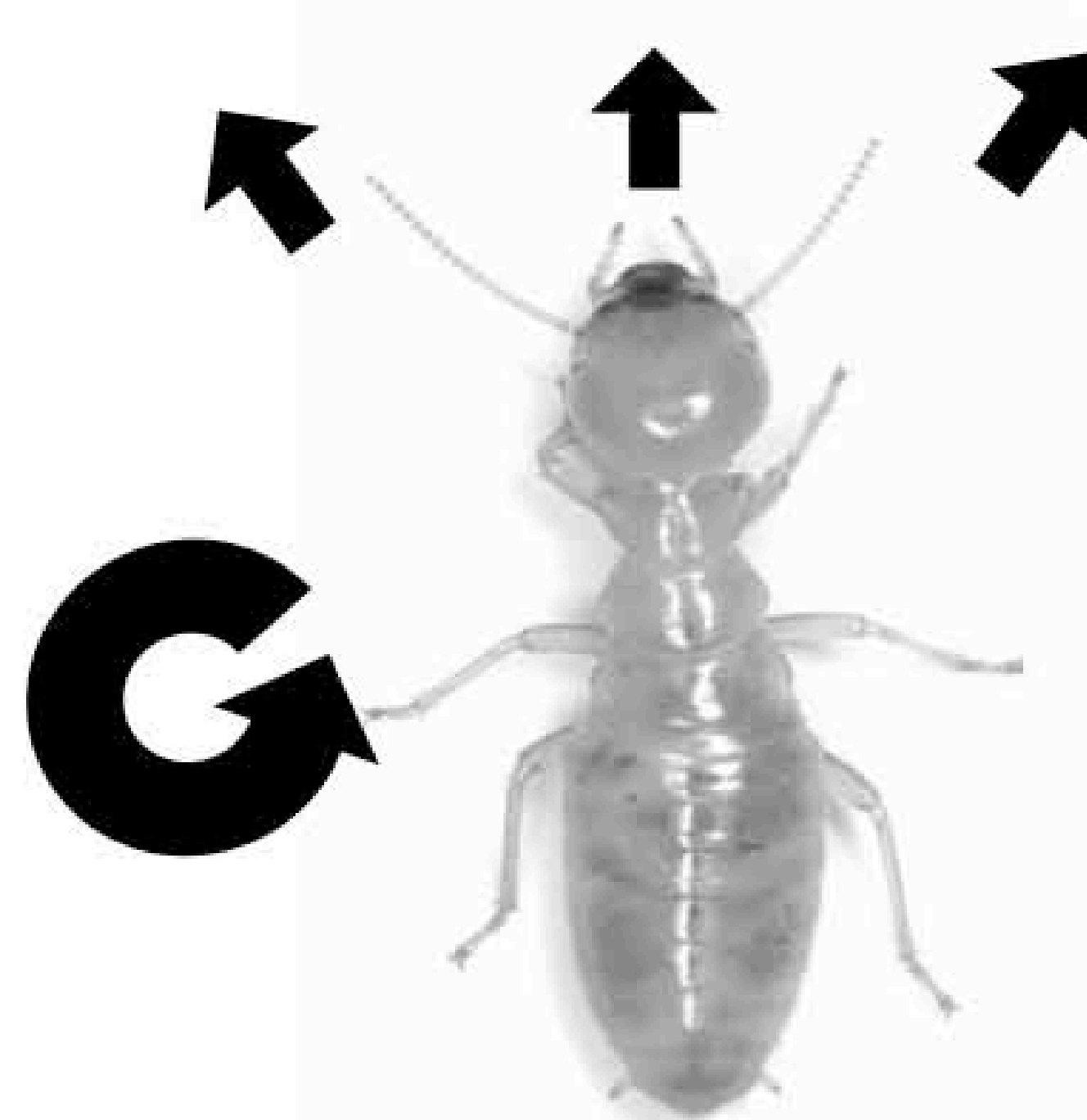

Pseudergate (Worker)

$\uparrow$ Moult

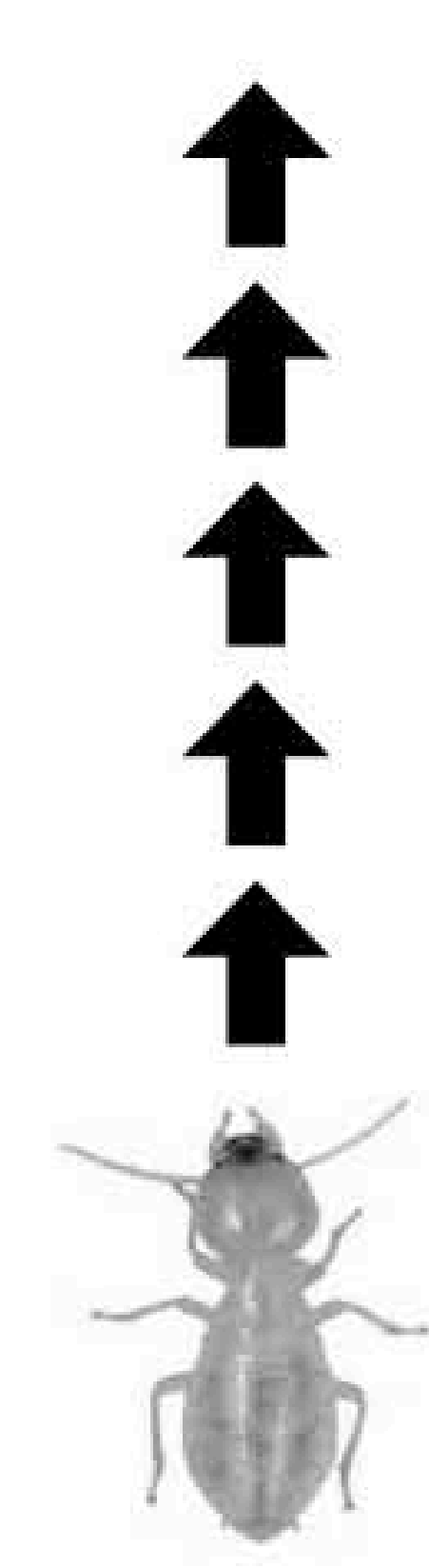

Egg (b)

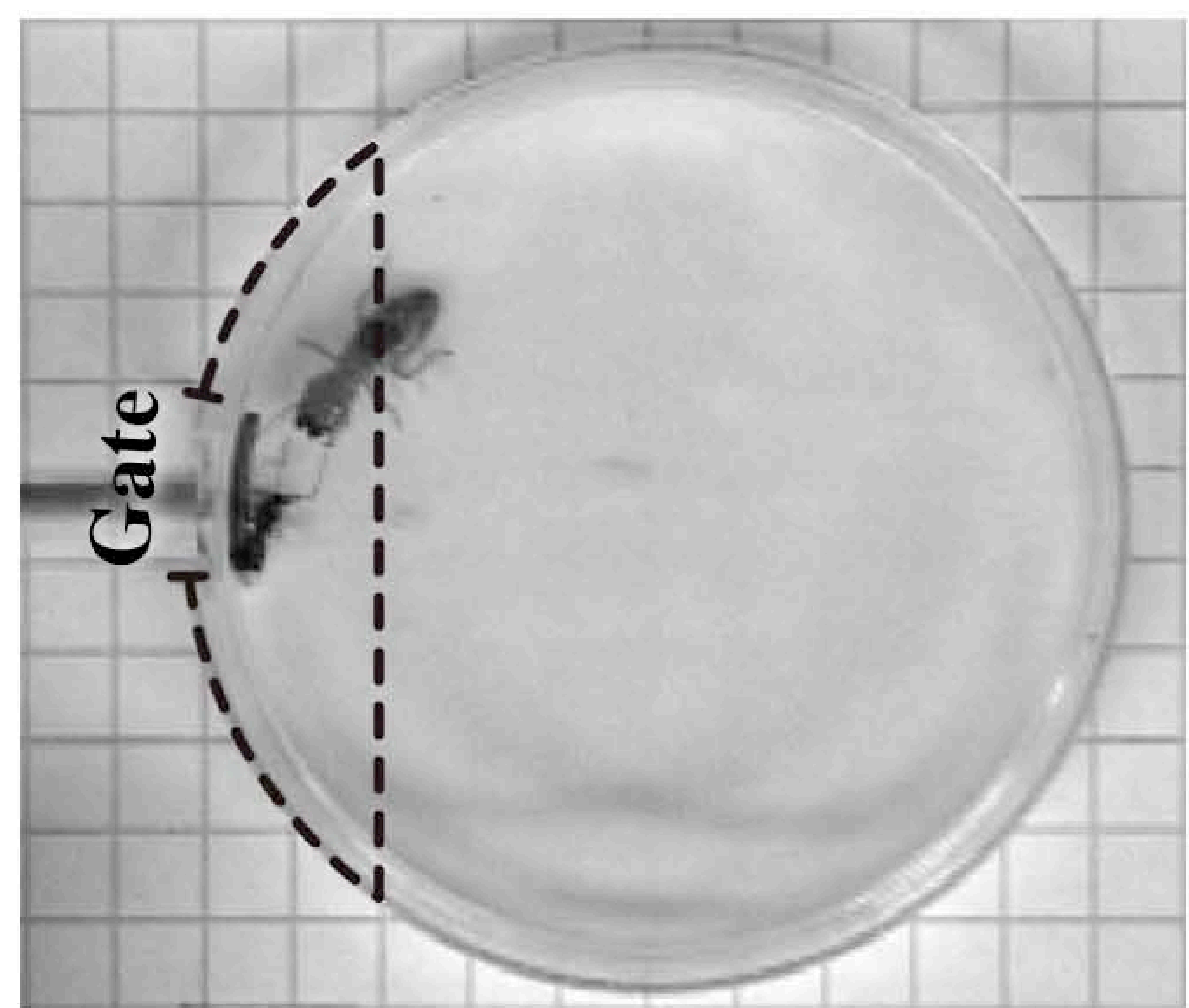

Enemy invasion under isolated condition

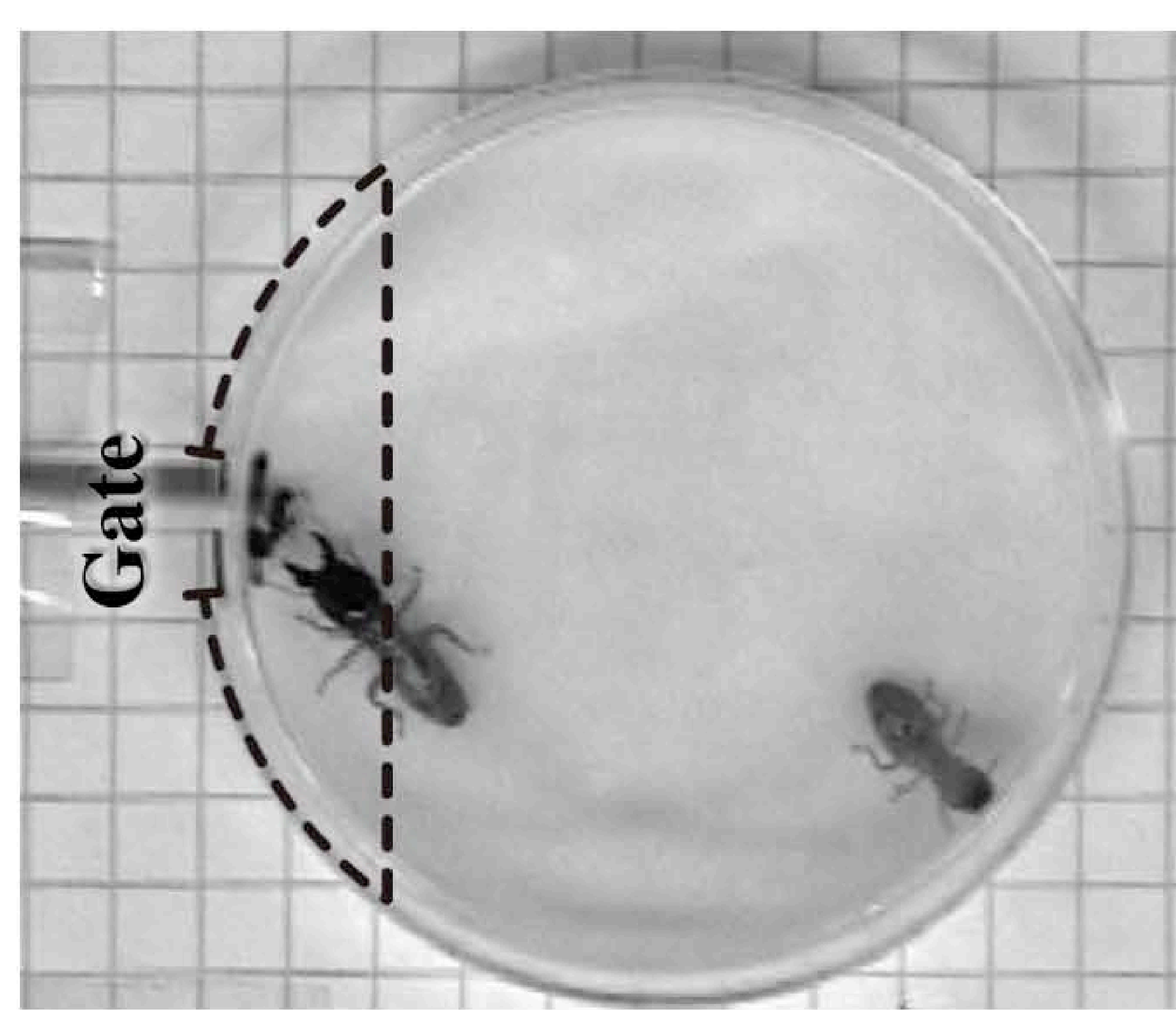

Enemy invasion under paired condition

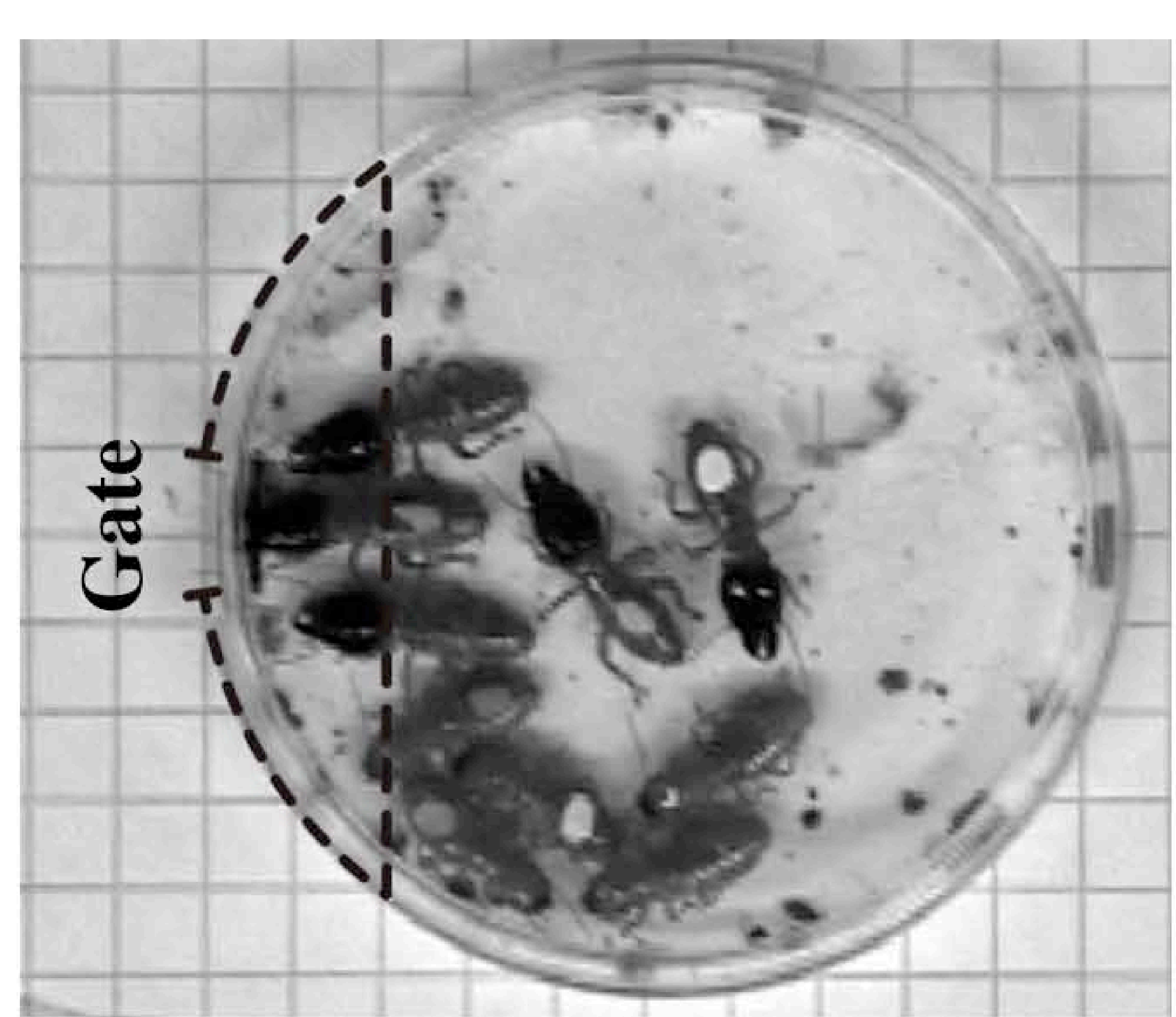

Nest opening 


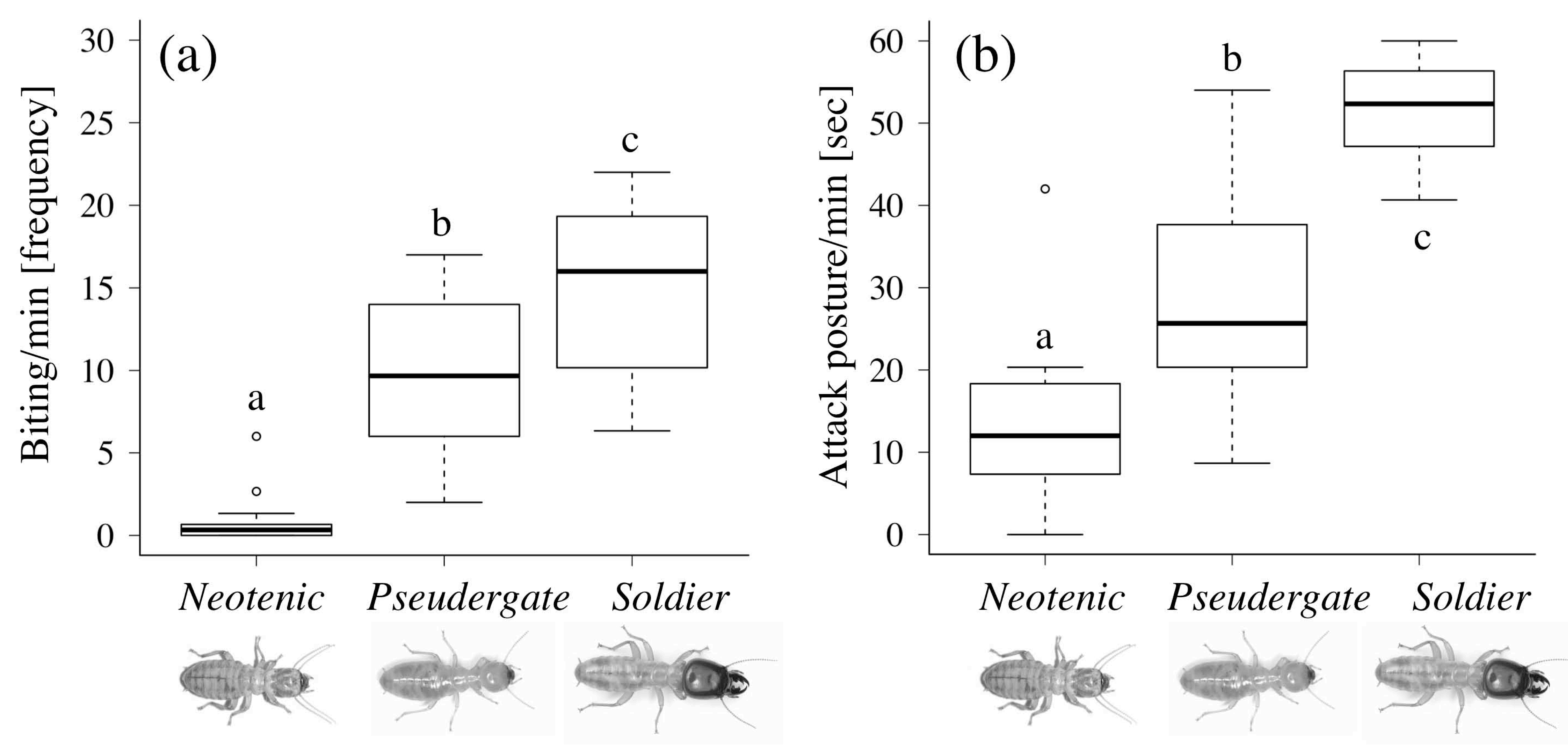

Figure 2 

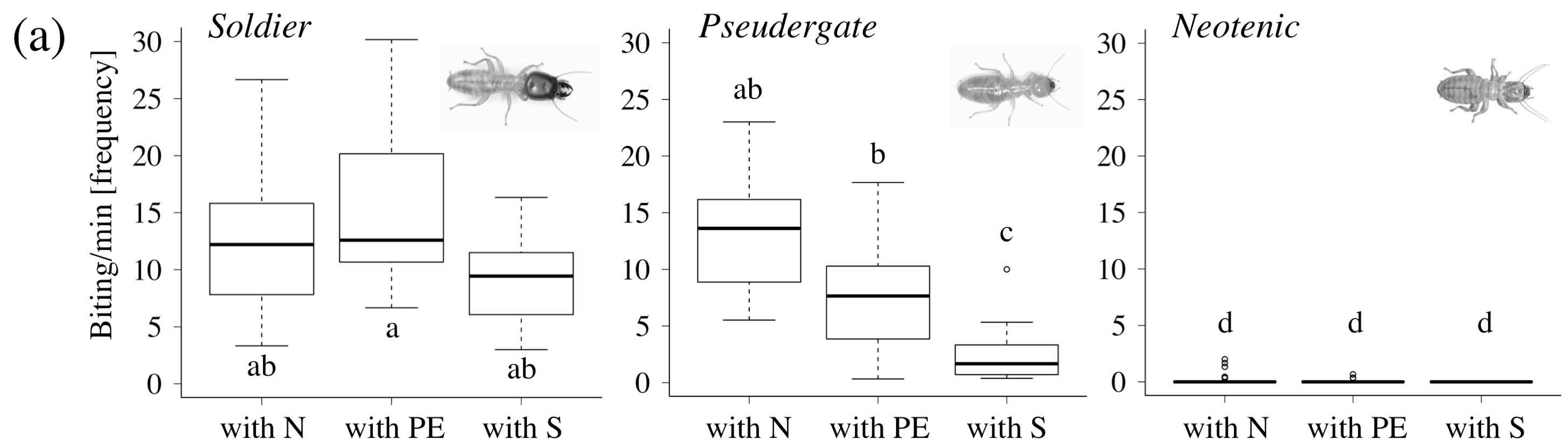

(b)

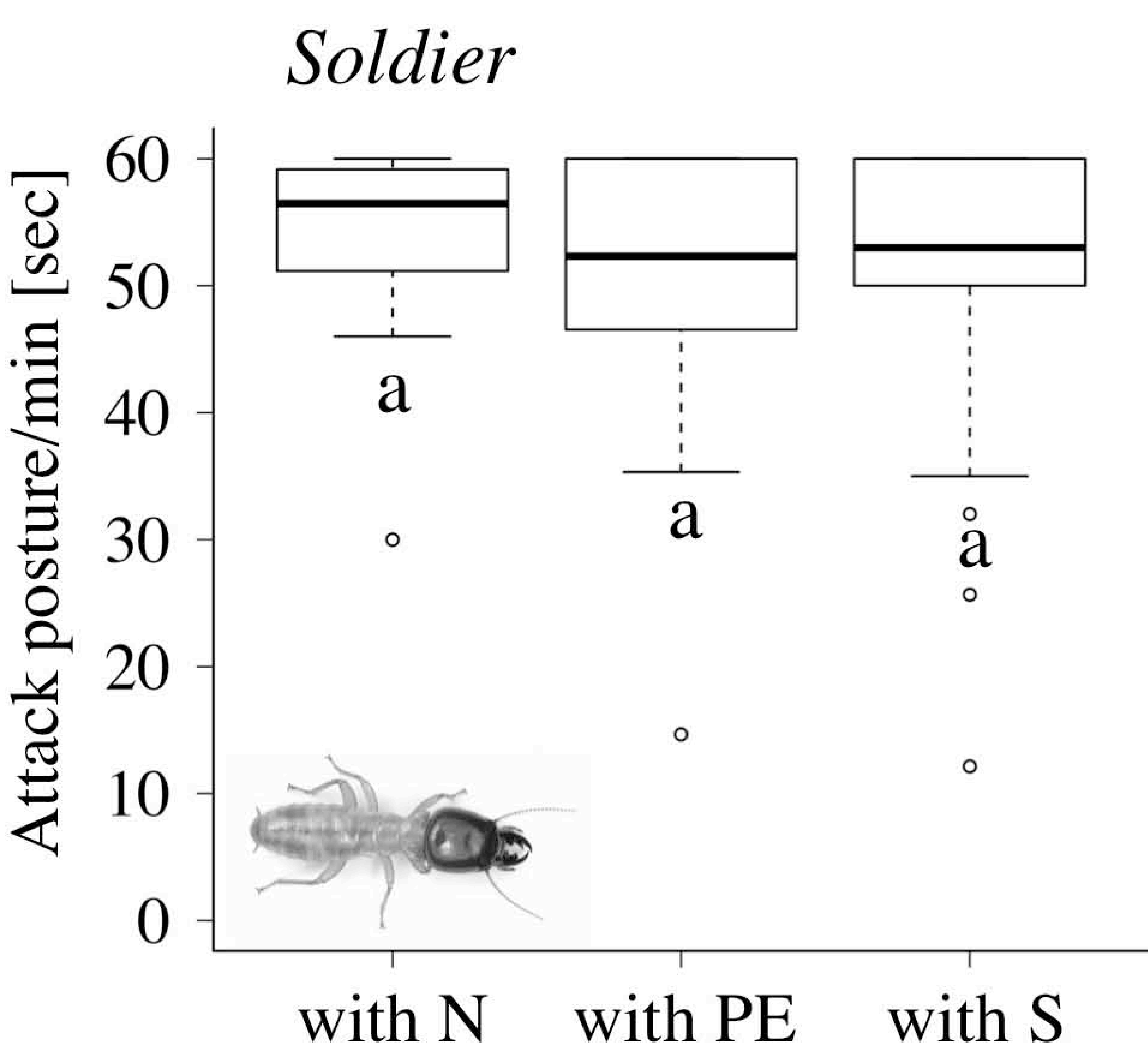

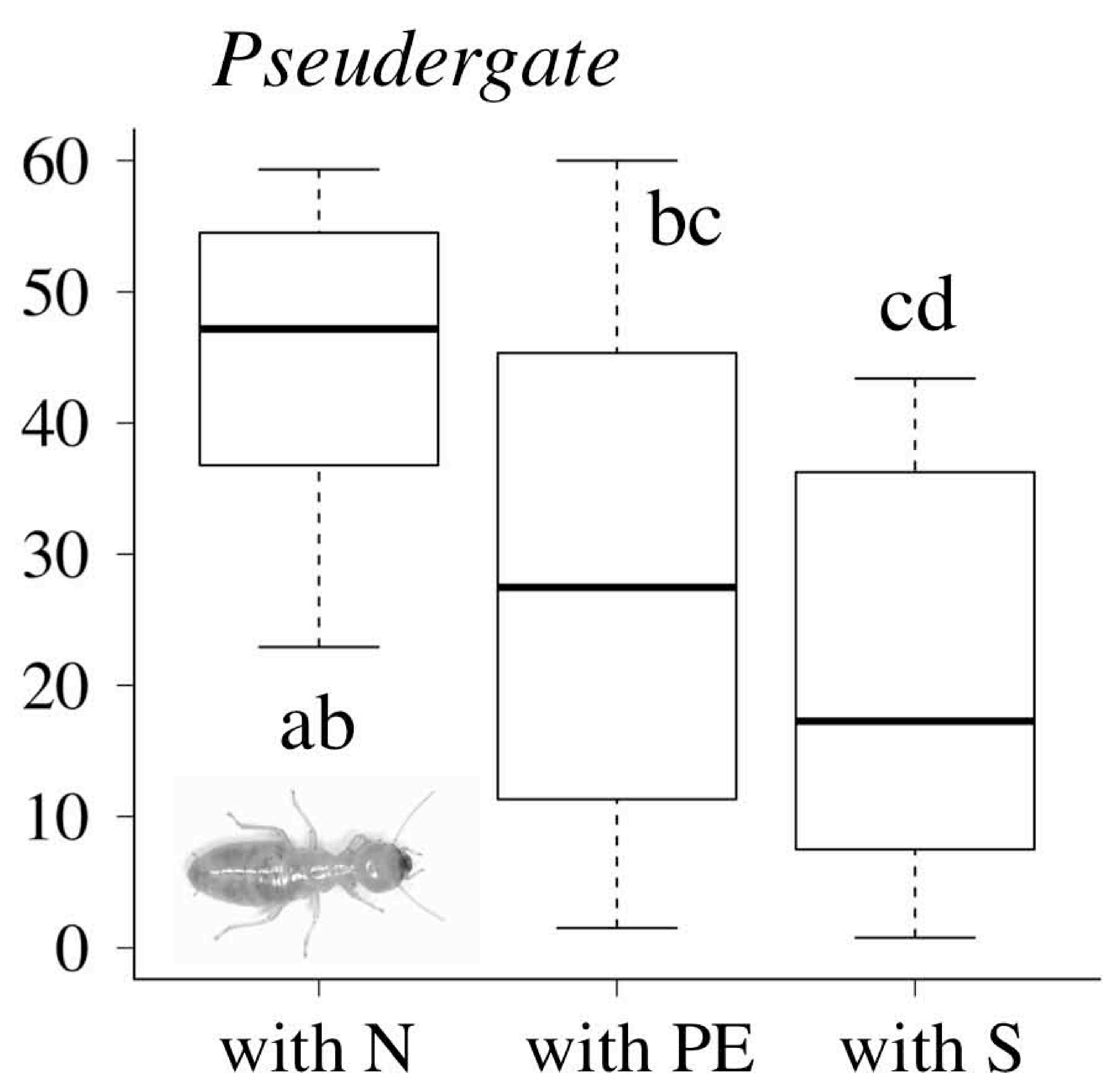

Figure 3 

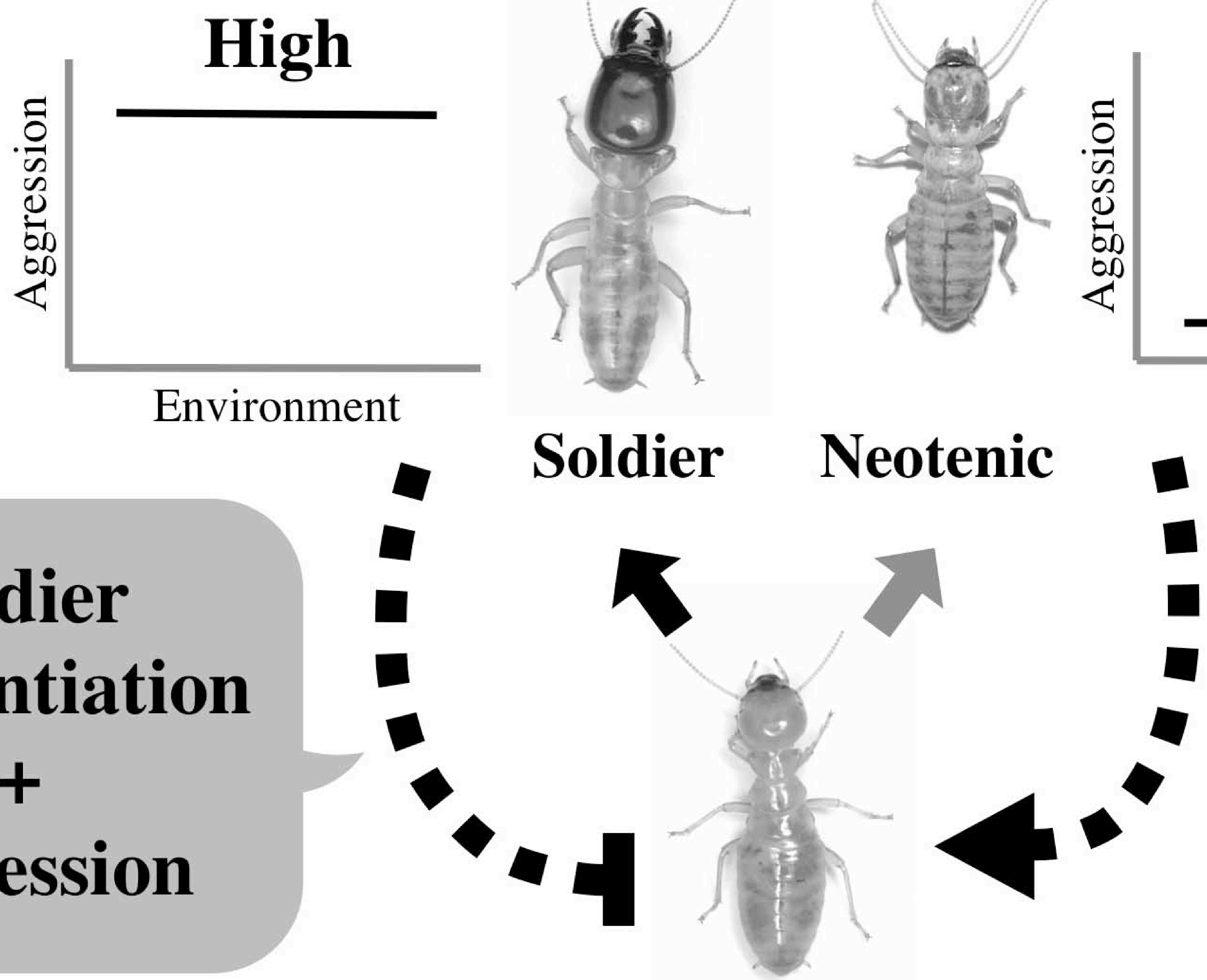

Pseudergate

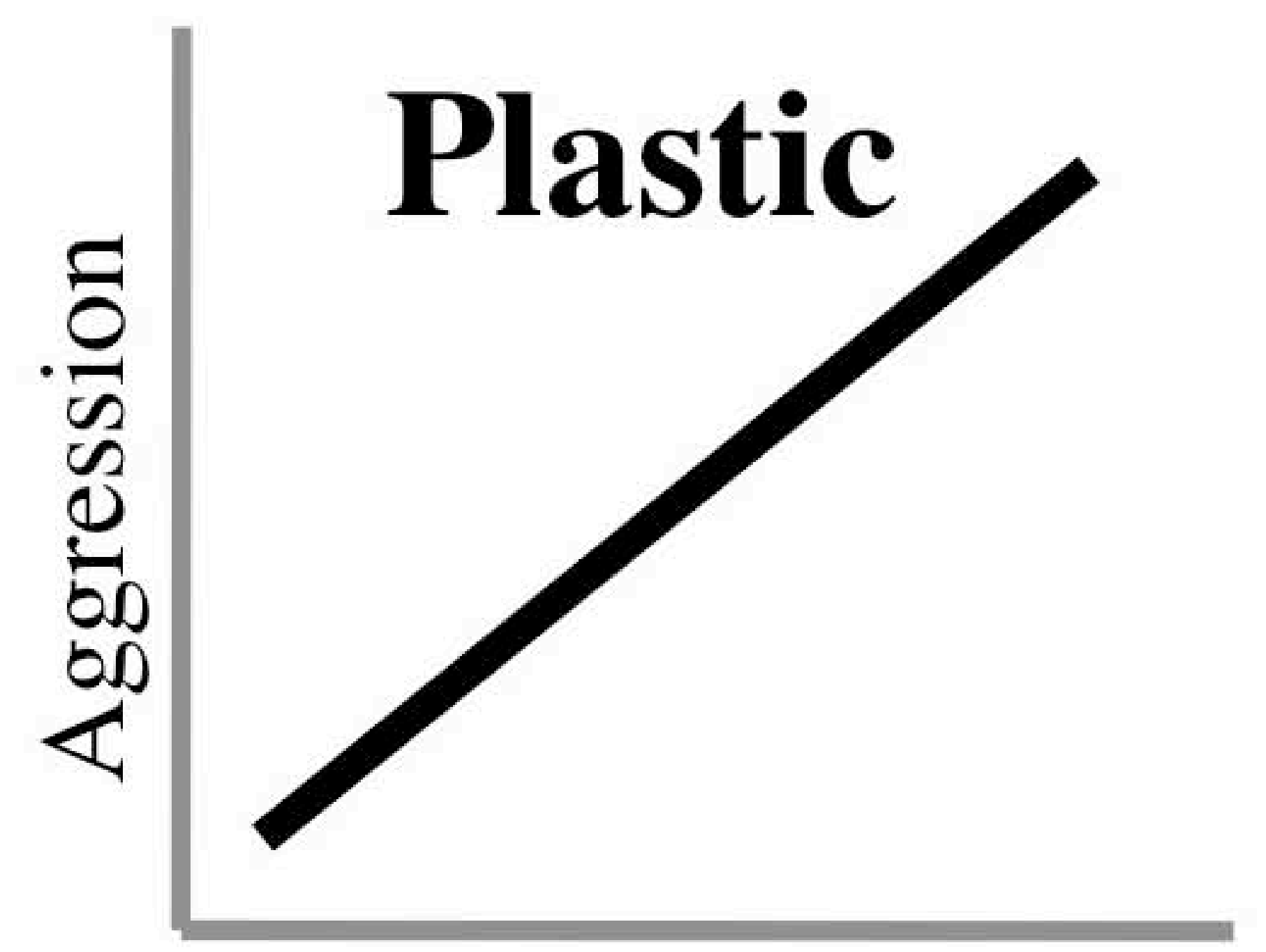

Environment
Environment

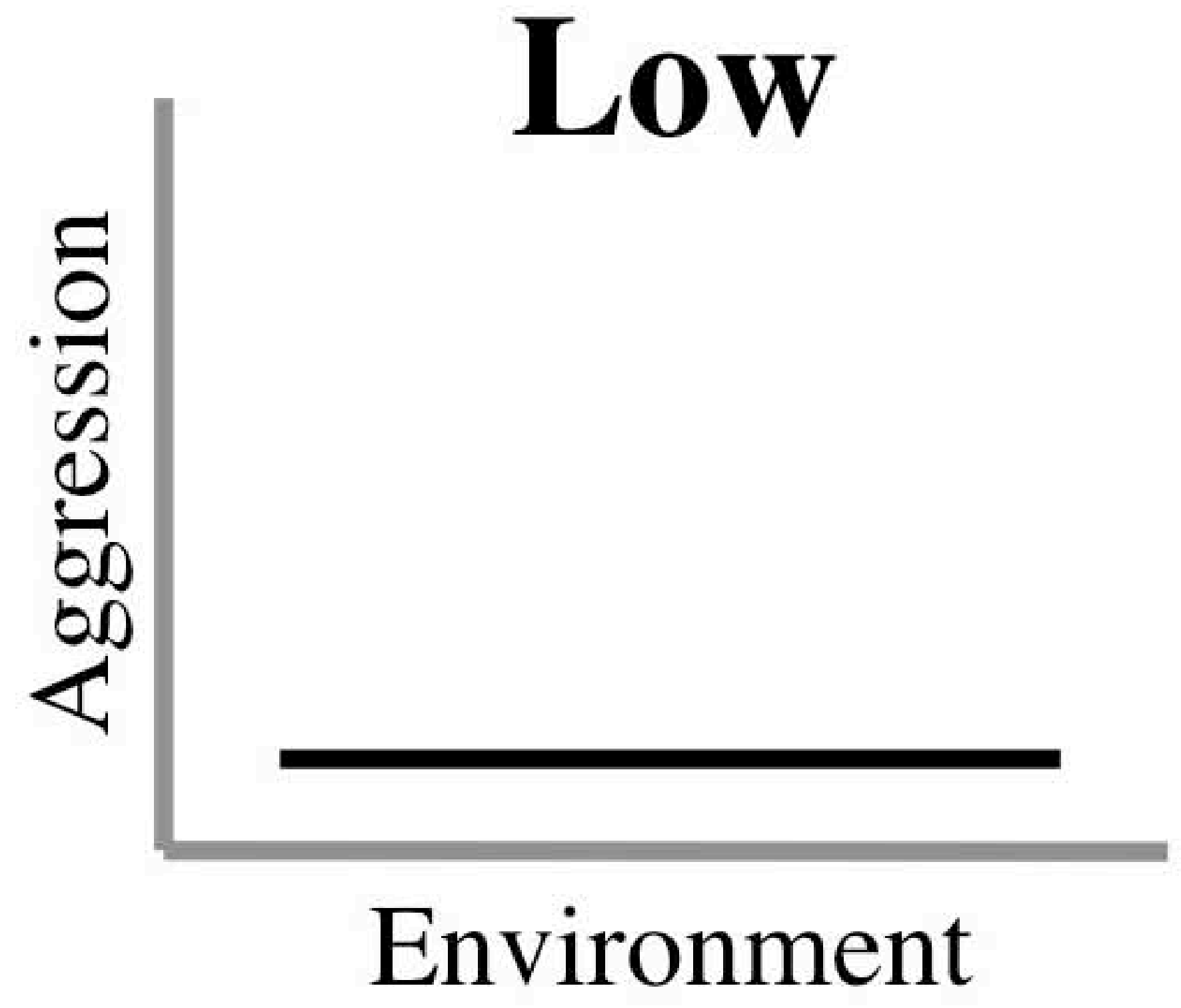

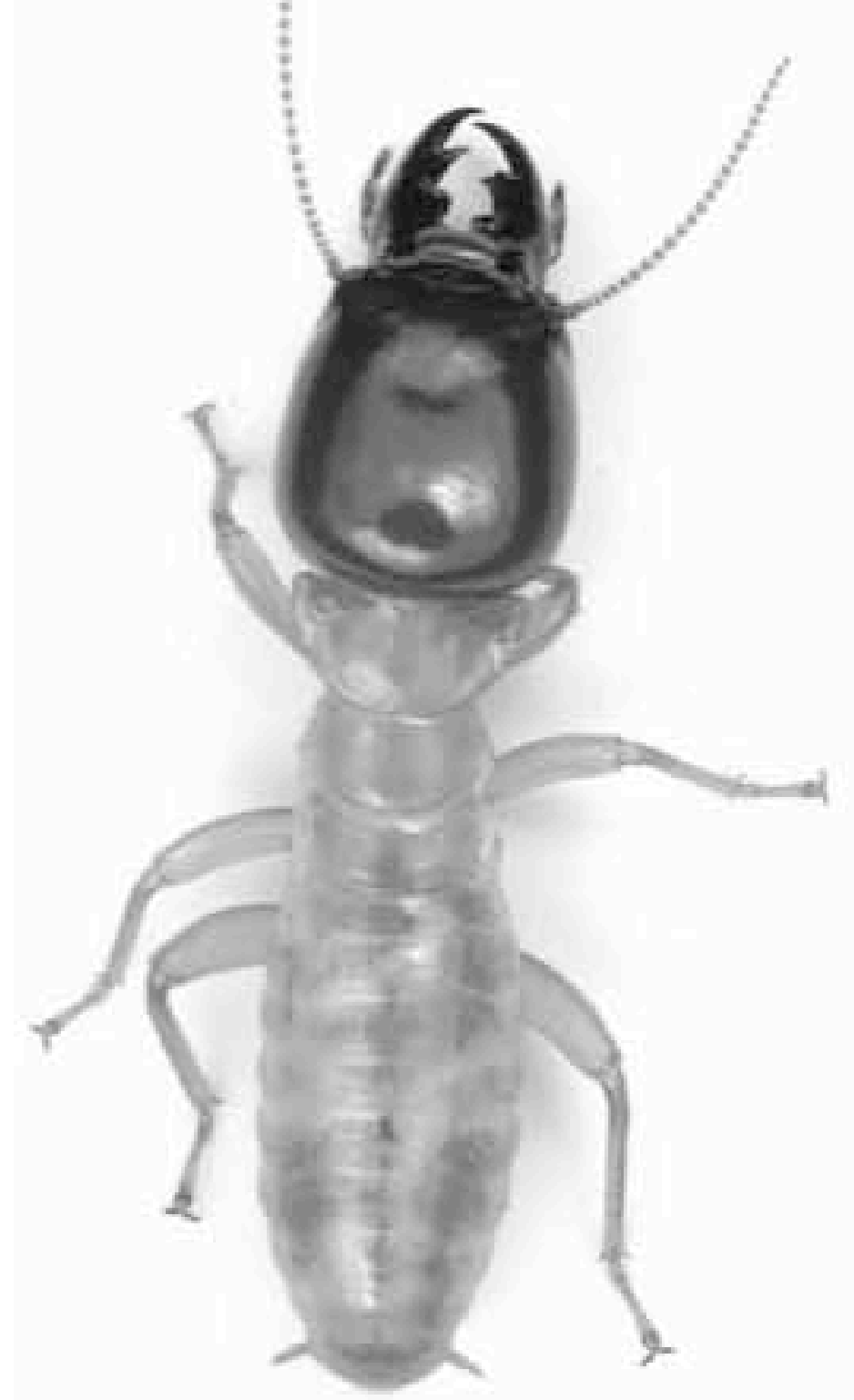

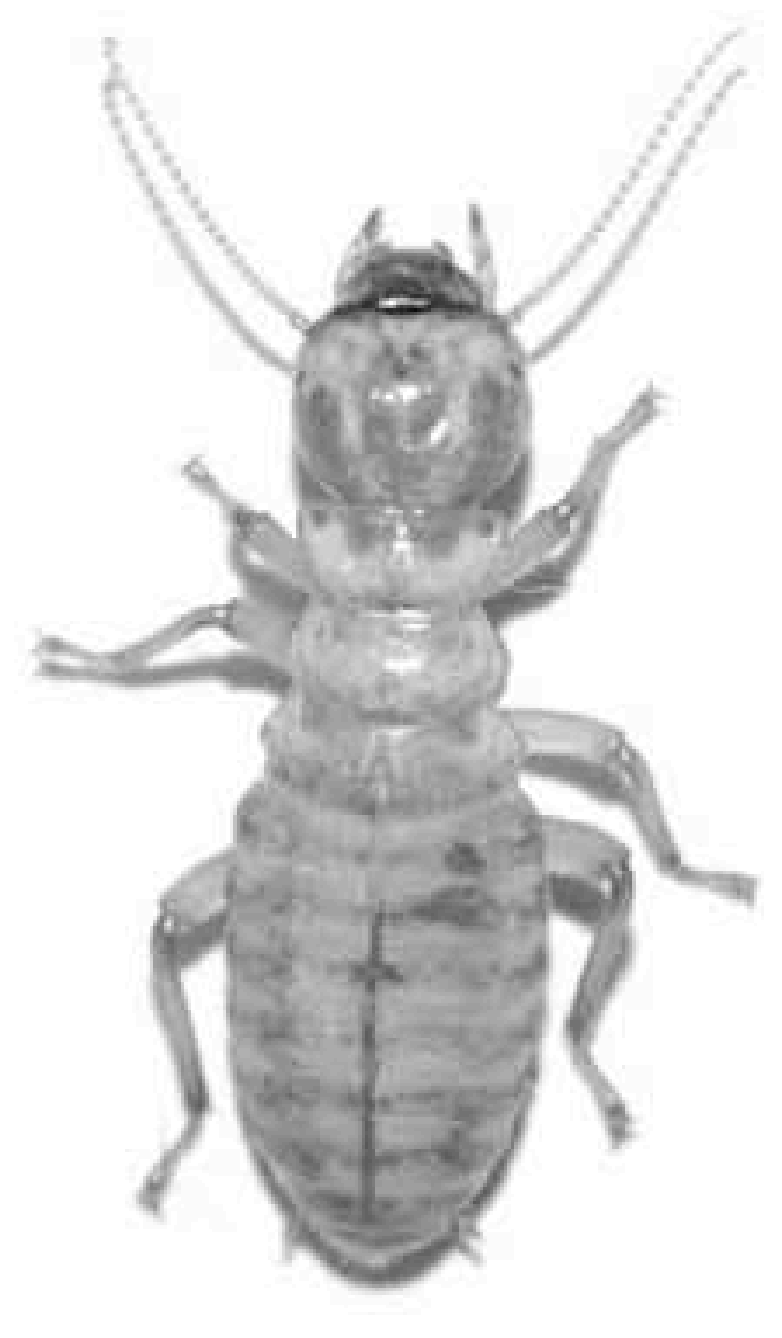

Environment

\section{Aggression \\ Soldier \\ differentiation \\ $+$}

